\title{
Clinical Applications of CT Myocardial Perfusion Imaging
}

pISSN 2508-707X / elSSN 2508-7088 https://doi.org/10.22468/cvia.2020.00094 Cardiovasc Imaging Asia 2020;4(4):86-90

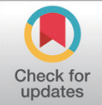

Received: October 14, 2020

Revised: October 27, 2020

Accepted: October 27, 2020

Corresponding author

Yining Wang, MD

Department of Radiology,

Peking Union Medical College Hospital,

Chinese Academy of Medical Sciences

and Peking Union Medical College,

No.1, Shuaifuyuan, Dongcheng District,

Beijing 100730, China

Tel: 86-010-69155509

Fax: 86-010-69155441

E-mail: yiningpumc@163.com

\author{
Cheng $\mathrm{Xu}$, Yan Yi, Yining Wang \\ Department of Radiology, Peking Union Medical College Hospital, \\ Chinese Academy of Medical Sciences and Peking Union Medical College, Beijing, China
}

With the continuous development of CT technology, myocardial CT perfusion imaging (CTP) has exhibited great advantages and been shown to have incremental value over coronary CT angiography, providing functional evaluation of coronary artery disease (CAD). Comprehensive assessment of CAD using cardiac CT has become feasible. This article introduces the preliminary clinical experience and advances with CTP and briefly reviews the feasibility verification, diagnostic performance, clinical strategy optimization and prognostic evaluation of CTP.

Key words Coronary artery disease $\cdot$ Tomography, X-ray computed Myocardial perfusion imaging.

\section{INTRODUCTION}

Coronary artery disease (CAD) remains a leading cause of mortality around the world and produces immense health and economic burdens globally. Coronary heart diseases accounted for $43.2 \%$ of cardiovascular disease mortality according to the American Heart Association heart disease and stroke statistics - 2019 update [1].

Comprehensive evaluation of CAD includes morphological and functional assessment [2]. Coronary CT angiography (CCTA) has been established as one of the first morphological evaluation technology options for excluding obstructive CAD that is associated with a high sensitivity and negative predictive value (NPV). The patient-based analysis of CCTA for detecting stenosis $\geq 50 \%$, according to invasive coronary angiography (ICA) revealed a sensitivity of $98.8 \%$, a specificity of $50 \%$, a positive predictive value (PPV) of $92.4 \%$, and a NPV of $87.5 \%$; the segment-based analysis revealed a sensitivity of $93.5 \%$, a specificity of $95 \%$, a PPV of $77.6 \%$, and an NPV of $98.7 \%$ [3]. However, such diagnoses are limited to the anatomical level and can overestimate the degree of stenosis. Direct revascularization based on vascular stenosis is not applicable to all CAD patients, as morphological and functional abnormalities do not match exactly

(c) This is an Open Access article distributed under the terms of the Creative Commons Attribution Non-Commercial License (https://creativecommons.org/licenses/by$\mathrm{nc} / 4.0$ ) which permits unrestricted non-commercial use, distribution, and reproduction in any medium, provided the original work is properly cited.
$[4,5]$. Therefore, a comprehensive evaluation of CAD is needed. In contrast, myocardial perfusion imaging (MPI) has been developed as an effective method for assessing the myocardial ischemia caused by CAD. MPI techniques mainly include positron emission tomography (PET), single photon emission tomography-MPI (SPECT-MPI), cardiac MR-MPI and CT-based MPI (CTP) [6-8]. There are advantages and limitations to both approaches. The traditional "gold standard" PET/SPECT-MPI has high sensitivity and diagnostic accuracy, but the limitations include high cost, attenuation artifacts, low spatial resolution, and poor sensitivity for tiny ischemia. MR-MPI is free of ionizing radiation and has high soft tissue contrast, but it requires a long examination time, is associated with complicated operations and has contraindications. In a meta-analysis [9], the sensitivity and specificity to detect CAD at a patient level were $84 \%$ and $87 \%$ for PET, $74 \%$ and $79 \%$ for SPECT, and $89 \%$ and $87 \%$ for MRI. The advantages of CTP include high spatial resolution, absolute quantitative analysis, and providing "one-stop" cardiac CT examination when combined with CCTA. The disadvantages to this approach include image artifacts and radiation exposure.

\section{PRELIMINARY CLINICAL EXPERIENCE AND PROGRESS}

To assess the preliminary clinical experience, Wang et al. [10] conducted a CTP study that included a group of 30 patients 
who went through both CCTA and adenosine stress CTP, in which myocardial perfusion reduction and more than moderate stenosis of lesions were analyzed. The reference standards were SPECT myocardial perfusion and ICA. Results demonstrated that adenosine stress CTP detected myocardial perfusion defects in good correlation with nuclear MPI. The results indicated that CTP combined with CCTA improves the diagnostic accuracy for identifying flow-obstructing stenosis compared with CCTA alone.

There are several limitations for this traditional CCTA and CTP study. Because the third-generation dual source CT (DSCT) tool is now available, the potential advancements for CT technology provide greater possibilities for extensive clinical utilization of CTP, and corresponding improvements have been made in other studies. A series of adenosine stress dynamic myocardial CTP studies were recently launched that used the third-generation DSCT. CT perfusion was performed using a prospective electrocardiogram-triggered table shuttle mode.

\section{Single-phase CCTA}

Advances in CT allow the coronary arterial tree to be captured entirely during one CTP scan. Yi et al. [11] investigated the potential value of single-phase coronary CT angiography (SP-CCTA) extracted from stress dynamic myocardial CT perfusion for coronary artery stenosis assessment. The image quality (IQ) and diagnostic value were compared using the coronary angiography (CAG) as a reference standard. A total of 56 patients underwent the CTP and CCTA examination, among which 39 patients underwent CAG. Results showed that the IQ and diagnostic value of SP-CCTA were equivalent to those of routine CCTA on third-generation DSCT. On a per-segment basis, the sensitivity, specificity, PPV, NPV, diagnostic accuracy and area under the receiver-operating-characteristic curve (AUC) results of SP-CCTA and routine CCTA for diagnosing stenosis $\geq 50 \%$ exhibited no significant difference (SP-CCTA: $78.1 \%, 94.9 \%, 77.4 \%, 95.1 \%, 91.6 \%$ and 0.935 vs. routine
CCTA: $74.7 \%, 95.3 \%, 78.0 \%, 95.3 \%, 91.6 \%$ and 0.937 ; all at a $\mathrm{p}$ value of 0.05$)$. SP-CCTA images from CTP could replace a separate routine CCTA, which would allow for the possibility of a "one-stop" cardiac examination for high-risk CAD patients who need myocardial ischemia assessment. Fig. 1 shows a case for SP-CCTA.

\section{Optimal parameter evaluation}

To date, the optimization of myocardial CT perfusion assessment remains inconsistent and uncertain. Yi et al. [12] conducted a serial study to explore a superior analysis selection process for myocardial blood flow (MBF) assessment on CTP for diagnosing hemodynamically significant CAD using fractional flow reserve (FFR) as a gold standard for assessing blood flow. For the relative MBF ratio [12], the calculation methods are nonuniform. In this study, a total of 151 vessels of 60 patients ( 43 males and 17 females; $61.38 \pm 8.01$ years) were enrolled in the analysis. The semiautomatic relative MBF ratio was calculated using the manually obtained semiautomatic absolute value and the reference MBF value, which was defined as the mean (ratio 1)/third quartile (ratio 2)/highest (ratio 3) automatic segmental value of 17 myocardial segments according to the following formula: relative $\mathrm{MBF}$ ratio=semiautomatic absolute $\mathrm{MBF}$ value/reference MBF value. All the data was derived from both the endocardial and transmural layers of the myocardium. The results showed that the relative MBF parameter of the endocardial myocardium that used the highest segmental MBF value as a reference provided optimal diagnostic accuracy for detecting hemodynamically significant CAD (AUC $=0.906,95 \%$ CI: 0.857-0.954). In further comparison [13], results showed that the absolute $\mathrm{MBF}$ value from the endocardial myocardium on stress dynamic myocardial CTP (AUC $=0.955,95 \%$ CI 0.9080.983), showed superior diagnostic performance compared to the relative MBF ratio for detecting myocardial ischemia in intermediate-to-high risk patients. The absolute MBF value provided an incremental benefit toward diagnostic performance of

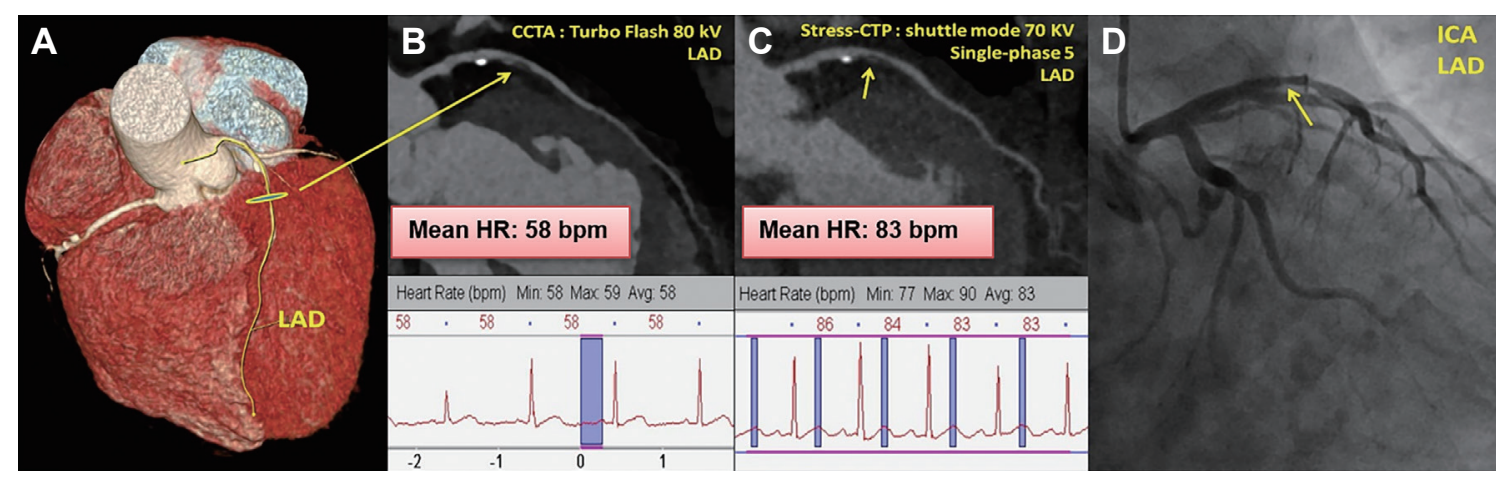

Fig. 1. Example case. Routine CCTA (A and B) shows a mixed plaque (arrow) in the proximal segments of the LAD branch. Single-phaseCCTA (C) also shows the lesion clearly (arrow). Stenosis of the lumen (arrow) is confirmed by the ICA (D). Mean HR during the routine CCTA was 58 bpm; however, mean HR during CTP was 83 bpm, which was significantly higher than routine CCTA. LAD: left anterior descending, ICA: invasive coronary angiography, CCTA: coronary CT angiography, CTP: CT-based myocardial perfusion imaging, HR: heart rate. 
the relative $\mathrm{MBF}$ ratio evaluation.

\section{Low dose "one-stop" CTP}

Furthermore, Yi et al. [14] conducted a study to investigate the diagnostic performance of $70-\mathrm{kVp}$ stress dynamic myocardial CT perfusion as a low-dose, one-stop cardiac CT examination in clinical application. Seventy-one consecutive symptomatic patients were prospectively recruited and scanned with stress dynamic myocardial CTP. The CTP phase with the best enhancement of the coronary arteries was selected and extracted as the CTP-derived SP-CCTA. The diagnostic performance of CTP and CTP+SP-CCTA for functionally significant CAD was assessed. ICA and FFR were used as the reference standard for the myocardial ischemia evaluation. The AUC of CTP+SPCCTA (AUC $=0.963$, 95\% CI: 0.938-0.989) was significantly superior to that of CTP (AUC $=0.922,95 \% \mathrm{CI}: 0.880-0.964)$ and that of SP-CCTA (AUC=0.833, 95\% CI: 0.765-0.900) alone (all $\mathrm{p}<$ 0.01). CTP-derived SP-CCTA improved the diagnostic value of CTP. Therefore, the innovative low-dose, one-stop CTP examination, which shows promising performance in terms of myocardial ischemia detection and low radiation dose, is clinically feasible for patients who require myocardial perfusion assessment.

\section{LITERATURE REVIEW AND FURTHER EXPECTATIONS}

Advancements in and further expectations for CTP consist of feasibility verification, diagnostic performance, clinical strategy optimization and prognosis evaluation.

\section{Feasibility verification}

Magalhães et al. [15] published a workflow for interpreting CCTA and myocardial CTP in the Journal of Cardiovascular Computed Tomography. Combining CCTA and myocardial CTP findings through a comprehensive protocol is feasible for identifying flow-limiting stenosis. The first step was CCTA interpretation, grading stenosis by visual assessment. When lumen analysis was compromised by artifacts, excessive calcification and/or stent with poor luminal assessment, the vessel was recorded as uninterpretable. Myocardial CTP assessment was performed using qualitative metrics, defined by the presence of any perfusion deficit as well as its reversibility. Finally, the readers combined anatomical and perfusion information on a visual basis, to match $\geq 50 \%$ stenosis with related fixed or reversible perfusion. Uninterpretable segments in CCTA that could be associated with reversible perfusion deficits were classified as "positive" for stenosis ( $\geq 50 \%$ stenosis). Additionally, readers were able to "reclassify" borderline or equivocal stenosis by CCTA, upgrading or downgrading lesions according to the findings of the myocardial CTP analysis.

\section{Diagnostic performance}

As for diagnostic accuracy of CTP, Li et al. [16] published their study in radiology, which investigated the diagnostic performance of dynamic CT MPI and machine learning-based CT-derived FFR (FFRct) for functional assessment of coronary stenosis. Eighty-six participants with 157 target vessels were included in the final analysis. Results showed that MBF derived from dynamic CT MPI outperformed machine learning-based CT fractional flow for identifying lesions causing ischemia (vessel-based analysis: $\mathrm{AUC}=0.96$ vs. $0.79, \mathrm{p}<0.001$; patient-based analysis: $\mathrm{AUC}=0.98$ vs. $0.85, \mathrm{p}<0.001)$. Pontone et al. [17] also proved that the inclusion of stress CTP for evaluating patients with an intermediate to high risk for CAD was feasible and improved the diagnostic performance of CCTA for detecting functionally significant CAD.

\section{Clinical strategy optimization}

To assess the clinical value of CTP, Pontone et al. [18] analyzed the diagnostic accuracy of CCTA alone or an integrated evaluation of CCTA+FFRct, CCTA+CTP, or CCTA+FFRct+CTP to detect functionally significant $\mathrm{CAD}$, using invasive evaluation as a reference standard. The study showed that both the addition of FFRct and CTP improved the AUC (0.876 and 0.878, respectively) as compared with CCTA alone $(0.826 ; \mathrm{p}<0.05)$. The sequential strategy of CCTA+FFRct+CTP showed the highest AUC (0.919; $\mathrm{p}<0.05)$, compared with all other strategies. The mean effective radiation dose for stress CTP was $5.3 \pm 0.7 \mathrm{mSv}$. Grandhi et al. [19] performed a feasibility study to assess direct costs and length of stay of a combined stress CTP/CCTA and used SPECT MPI as a benchmark in chest pain patients at intermediate-risk for acute coronary syndrome presenting to the emergency department. Results showed the median length of stay and direct cost incurred by patients who underwent combined stress CTP/CCTA were shorter and lower than those who underwent SPECT-MPI, with the direct costs being $44 \%$ less, which was significantly lower. Baggiano et al. [20] assessed the impact of CTP availability on managing patients with suspected CAD that were scheduled for ICA and invasive FFR. Results showed that the rate of reclassification of patients with CTP was $34 \%$ (100 out of 291 patients), the rate of agreement with the final treatment decision was $89 \%$ (261 out of 291 patients) and the rate of agreement on vessels to be revascularized was 74\% (216 out of 291 patients).

A single-center study demonstrated that selective use of CT MPI improves hemodynamic classification for this range of intermediate FFRct results [21]. A meta-analysis [22] has proven that CTP has better diagnostic accuracy for obstructive stenosis than CCTA at the vessel level (AUC: 0.911 vs. 0.787). The multi-center CRESCENT II study [23] tested a tiered comprehensive cardiac CT protocol, including a calcium score scan, 
CCTA, and stress CTP, that would allow safe exclusion of CAD by relatively simple means while at the same time incorporating functional measures of CAD for well-informed decisions and avoiding premature invasive procedures. The results showed that further testing was needed in $13 \%$ of patients randomized to CT, compared with $37 \%$ after functional testing. The combined CT protocol achieved a diagnosis more rapidly, and it removed the need for additional noninvasive testing. In another meta-analysis, Siontis et al. [24] evaluated differences in downstream testing, coronary revascularization, and clinical outcomes, following non-invasive diagnostic modalities used to detect CAD. The results showed that an initial diagnostic strategy of stress perfusion imaging is associated with fewer referrals for ICA and revascularization procedures than non-invasive anatomical testing.

\section{Prognosis evaluation}

As for prognostic the value of CTP, Meinel et al. [25,26] investigated the prognostic value of myocardial perfusion CT for major adverse cardiac events. Data for 144 patients from six centers who had undergone CCTA and CT-MPI were assessed, and the results showed that global quantification of left ventricular MBF at stress dynamic CT-MPI may have an incremental predictive value for future major adverse cardiovascular events over clinical risk factors and assessing stenosis at CCTA. Nakamura et al. [27] proved that stress dynamic CTP with CT delayed-enhancement had incremental prognostic value over CT angiography in each group with suspected or known CAD, and was prognostically useful in subgroups of patients with stent, heavy calcification, or obstructive CAD. Ischemic perfusion defects and myocardial scarring may play complementary roles in prognostic stratification.

\section{CTP EXPERT CONSENSUS AND COST-EFFECTIVENESS ANALYSIS}

In 2020, the Society of Cardiovascular Computed Tomography published an expert consensus on myocardial computed tomography perfusion imaging [28], providing a consensus document that can be used as a resource for clinical implementation, resource allocation, and future investigations. As for CTP cost-effectiveness analysis, Kim et al. [29] performed a study to determine the cost-effectiveness of stress CTP, CCTA, and the combination of both in suspected obstructive CAD or in-stent restenosis (ISR) in patients with previous coronary stent implantation. The cost-effectiveness was assessed based on a cost-effectiveness threshold of $\$ 100000$ per quality-adjusted life years. The results showed that CTP was cost-effective for detecting obstructive CAD or ISR in patients with previous stenting and therefore should be considered a feasible approach in daily clinical practice.

\section{CONCLUSION}

There is increasing evidence that myocardial CTP has great advantages and applications. Low-dose "one-stop" cardiac CT has become feasible for comprehensive evaluation of CAD. Further multi-center trails are needed to investigate the advantages of CTP and additional cost-effectiveness analyses are needed to further improve approaches for optimizing patient outcomes.

\section{Conflicts of Interest}

The authors have no potential conflicts of interest to disclose.

\section{Acknowledgments}

None.

\section{ORCID iDs}

$\begin{array}{ll}\text { Cheng Xu } & \text { https://orcid.org/0000-0003-4056-8082 } \\ \text { Yan Yi } & \text { https://orcid.org/0000-0003-2639-8574 } \\ \text { Yining Wang } & \text { https://orcid.org/0000-0001-6441-2002 }\end{array}$

\section{Author Contributions}

Conceptualization: Yining Wang. Investigation: Yan Yi, Cheng Xu. Validation: Yining Wang. Writing_original draft: Cheng Xu. Writing_review \& editing: Yan Yi, Yining Wang.

\section{REFERENCES}

1. Benjamin EJ, Muntner P, Alonso A, Bittencourt MS, Callaway CW, Carson AP, et al. Heart disease and stroke statistics-2019 update: a report from the American Heart Association. Circulation 2019;139:e56-e528.

2. Qayyum AA, Kastrup J. Measuring myocardial perfusion: the role of PET, MRI and CT. Clin Radiol 2015;70:576-584.

3. Chao SP, Law WY, Kuo CJ, Hung HF, Cheng JJ, Lo HM, et al. The diagnostic accuracy of 256-row computed tomographic angiography compared with invasive coronary angiography in patients with suspected coronary artery disease. Eur Heart J 2010;31:1916-1923.

4. Patel MR, Peterson ED, Dai D, Brennan JM, Redberg RF, Anderson HV, et al. Low diagnostic yield of elective coronary angiography. N Engl J Med 2010;362:886-895.

5. Tonino PA, Fearon WF, De Bruyne B, Oldroyd KG, Leesar MA, Ver Lee $\mathrm{PN}$, et al. Angiographic versus functional severity of coronary artery stenoses in the FAME study fractional flow reserve versus angiography in multivessel evaluation. J Am Coll Cardiol 2010;55:2816-2821.

6. Rochitte CE, George RT, Chen MY, Arbab-Zadeh A, Dewey M, Miller JM, et al. Computed tomography angiography and perfusion to assess coronary artery stenosis causing perfusion defects by single photon emission computed tomography: the CORE320 study. Eur Heart J 2014;35:11201130 .

7. George RT, Arbab-Zadeh A, Cerci RJ, Vavere AL, Kitagawa K, Dewey M, et al. Diagnostic performance of combined noninvasive coronary angiography and myocardial perfusion imaging using 320-MDCT: the CT angiography and perfusion methods of the CORE320 multicenter multinational diagnostic study. AJR Am J Roentgenol 2011;197:829-837.

8. Rossi A, Merkus D, Klotz E, Mollet N, de Feyter PJ, Krestin GP. Stress myocardial perfusion: imaging with multidetector CT. Radiology 2014;270: 25-46.

9. Takx RA, Blomberg BA, El Aidi H, Habets J, de Jong PA, Nagel E, et al. Diagnostic accuracy of stress myocardial perfusion imaging compared to invasive coronary angiography with fractional flow reserve meta-analy- 
sis. Circ Cardiovasc Imaging 2015;8:e002666.

10. Wang Y, Qin L, Shi X, Zeng Y, Jing H, Schoepf UJ, et al. Adenosine-stress dynamic myocardial perfusion imaging with second-generation dualsource CT: comparison with conventional catheter coronary angiography and SPECT nuclear myocardial perfusion imaging. AJR Am J Roentgenol 2012;198:521-529.

11. Yi Y, Wu W, Lin L, Zhang HZ, Qian H, Shen ZJ, et al. Single-phase coronary artery CT angiography extracted from stress dynamic myocardial CT perfusion on third-generation dual-source CT: validation by coronary angiography. Int J Cardiol 2018;269:343-349.

12. Yi Y, Xu C, Wu W, Wang Y, Li YM, Shen ZJ, et al. Myocardial blood flow analysis of stress dynamic myocardial CT perfusion for hemodynamically significant coronary artery disease diagnosis: the clinical value of relative parameter optimization. J Cardiovasc Comput Tomogr 2020;14:314-321.

13. Yi Y, Xu C, Wu W, Wang Y, Li YM, Ge YQ, et al. Stress dynamic myocardial CT perfusion for symptomatic patients with intermediate- or highrisk of coronary artery disease: optimization and incremental improvement between the absolute and relative myocardial blood flow analysis. J Cardiovasc Comput Tomogr 2020;14:437-443.

14. Yi Y, Xu C, Wu W, Shen ZJ, Lee W, Yun CH, et al. Low-dose CT perfusion with combined use of CTP and CTP-derived coronary CT angiography at $70 \mathrm{kVp}$ : validation with invasive fractional flow reserve. Eur Radiol 2020 Aug 18 [Epub]. http:/doi.org/10.1007/s00330-020-07096-x.

15. Magalhães TA, Kishi S, George RT, Arbab-Zadeh A, Vavere AL, Cox C, et al. Combined coronary angiography and myocardial perfusion by computed tomography in the identification of flow-limiting stenosis-The CORE320 study: an integrated analysis of CT coronary angiography and myocardial perfusion. J Cardiovasc Comput Tomogr 2015;9:438-445.

16. Li Y, Yu M, Dai X, Lu Z, Shen C, Wang Y, et al. Detection of hemodynamically significant coronary stenosis: CT myocardial perfusion versus machine learning CT fractional flow reserve. Radiology 2019;293:305-314.

17. Pontone G, Andreini D, Guaricci AI, Baggiano A, Fazzari F, Guglielmo M, et al. Incremental diagnostic value of stress computed tomography myocardial perfusion with whole-heart coverage CT scanner in intermediateto high-risk symptomatic patients suspected of coronary artery disease. JACC Cardiovasc Imaging 2019;12:338-349.

18. Pontone G, Baggiano A, Andreini D, Guaricci AI, Guglielmo M, Muscogiuri $\mathrm{G}$, et al. Dynamic stress computed tomography perfusion with a whole-heart coverage scanner in addition to coronary computed tomography angiography and fractional flow reserve computed tomography derived. JACC Cardiovasc Imaging 2019;12:2460-2471.

19. Grandhi GR, Batlle JC, Maroules CD, Janowitz W, Peña CS, Ziffer JA, et al. Combined stress myocardial CT perfusion and coronary CT angiogra- phy as a feasible strategy among patients presenting with acute chest pain to the emergency department. J Cardiovasc Comput Tomogr 2020 Jul 29 [Epub]. http://doi.org/10.1016/j.jcct.2020.06.195.

20. Baggiano A, Fusini L, Del Torto A, Vivona P, Guglielmo M, Muscogiuri G, et al. Sequential strategy including FFRCT plus stress-CTP impacts on management of patients with stable chest pain: The Stress-CTP RIPCORD Study. J Clin Med 2020;9:2147.

21. Coenen A, Rossi A, Lubbers MM, Kurata A, Kono AK, Chelu RG, et al. Integrating CT myocardial perfusion and CT-FFR in the work-up of coronary artery disease. JACC Cardiovasc Imaging 2017;10:760-770.

22. Lu M, Wang S, Sirajuddin A, Arai AE, Zhao S. Dynamic stress computed tomography myocardial perfusion for detecting myocardial ischemia: a systematic review and meta-analysis. Int J Cardiol 2018;258:325-331.

23. Lubbers M, Coenen A, Kofflard M, Bruning T, Kietselaer B, Galema T, et al. Comprehensive cardiac CT with myocardial perfusion imaging versus functional testing in suspected coronary artery disease: the multicenter, randomized CRESCENT-II trial. JACC Cardiovasc Imaging 2018;11:16251636.

24. Siontis GC, Mavridis D, Greenwood JP, Coles B, Nikolakopoulou A, Jüni $\mathrm{P}$, et al. Outcomes of non-invasive diagnostic modalities for the detection of coronary artery disease: network meta-analysis of diagnostic randomised controlled trials. BMJ 2018;360:k504.

25. Meinel FG, Wichmann JL, Schoepf UJ, Pugliese F, Ebersberger U, Lo GG, et al. Global quantification of left ventricular myocardial perfusion at dynamic CT imaging: prognostic value. J Cardiovasc Comput Tomogr 2017; 11:16-24.

26. Meinel FG, Pugliese F, Schoepf UJ, Ebersberger U, Wichmann JL, Lo GG, et al. Prognostic value of stress dynamic myocardial perfusion CT in a multicenter population with known or suspected coronary artery disease. AJR Am J Roentgenol 2017;208:761-769.

27. Nakamura S, Kitagawa K, Goto Y, Takafuji M, Nakamori S, Kurita T, et al. Prognostic value of stress dynamic computed tomography perfusion with computed tomography delayed enhancement. JACC Cardiovasc Imaging 2020;13:1721-1734.

28. Patel AR, Bamberg F, Branch K, Carrascosa P, Chen M, Cury RC, et al. Society of cardiovascular computed tomography expert consensus document on myocardial computed tomography perfusion imaging. J Cardiovasc Comput Tomogr 2020;14:87-100.

29. Kim SH, Rübenthaler J, Nörenberg D, Huber T, Kunz WG, Sommer WH, et al. Cost-effectiveness of stress CTP versus CTA in detecting obstructive CAD or in-stent restenosis in stented patients. Eur Radiol 2020 Sep 3 [Epub]. https://doi.org/10.1007/s00330-020-07202-z. 